\title{
Light Activation of the Phosphoinositide Cycle in Intrinsically Photosensitive Chicken Retinal Ganglion Cells
}

\author{
Maria Ana Contín, ${ }^{1}$ Daniela M. Verra, ${ }^{1}$ Gabriela Salvador, ${ }^{2}$ Monica Ilincheta, ${ }^{2}$ \\ Norma M. Giusto, ${ }^{2}$ and Mario E. Guido ${ }^{1}$
}

Purpose. In vertebrates, intrinsically photosensitive retinal ganglion cells (ipRGCs) acting as nonvisual photoreceptors transmit environmental illumination information to the brain, regulating diverse non-image-forming tasks. The phototransduction cascade in chicken ipRGCs has been shown to resemble that of rhabdomeric photoreceptors and involves phospholipase C (PLC) activation. The current work was an investigation of the participation of the phosphoinositide (PIP) cycle in this mechanism and of whether changes in activities of inositol 1,4,5-trisphosphate $\left(\mathrm{IP}_{3}\right)$ and PIP kinase are triggered by light.

Methods. Primary cultures of Thy-1 immunopurified chicken embryonic RGCs were exposed to bright light pulses or kept in the dark, to assess intracellular $\mathrm{Ca}^{2+}$ mobilization by Fluo-3 AM fluorescence microscopy, $\mathrm{IP}_{3}$ levels, and enzymatic activities of diacylglycerol, phosphatidylinositol, and phosphatidylinositol phosphate kinases (DAGK, PIK, and PIPK, respectively), by radioactive assays. The presence of different melanopsins (Opn $4 \mathrm{~m}$ and Opn4x) and other photopigments was determined by RT-PCR and immunochemistry.

Results. Cultured RGCs expressing different nonvisual photopigments displayed a significant and rapid increase in $\mathrm{IP}_{3}$ levels (1.3-fold) and $\mathrm{Ca}^{2+}$ mobilization by light, which was reversed by administration of the PLC inhibitor U73122 (5 $\mu \mathrm{M})$. Brief light pulses also caused a very rapid and transient activation of DAGK, PIK, and PIPK compared with that in the dark control.

Conclusions. The results indicate for the first time that light stimulation of chicken RGC cultures activates the PIP cycle, causing an increase in intracellular levels of $\mathrm{IP}_{3}$, changes in levels of phosphatidic acid, PIP, and $\mathrm{PIP}_{2}$; and mobilization

From the ${ }^{1} \mathrm{CIQUIBIC}$ (Centro de Investigaciones en Química Biológica de Córdoba) (CONICET; Consejo Nacional de Investigaciones Científicas y Técnicas), Departamento de Química Biológica, Facultad de Ciencias Químicas, Universidad Nacional de Córdoba-Córdoba; and ${ }^{2}$ INIBIBB (Instituto de Investigaciones Bioquímicas de Bahía Blanca), Universidad Nacional del Sur, Bahía Blanca, Argentina.

Supported by Agencia Nacional de Promoción Científica y Técnica (FONCyT, PICT 2004 No. 967 and PICT 2005 No. 31971), CONICET, SeCyT (Secretaria de Ciencia y Tecnología-UNC (Universidad Nacional de Córdoba), SeCyT-UNS (Universidad Nacional del Sur), Fundación Florencio Fiorini (2007), and MinCyT (Ministerio de Ciencia y Tecnología) Córdoba. MEG is a fellow of the John Simon Guggenheim Memorial Foundation, 2009.

Submitted for publication April 4, 2010; revised April 29, 2010; accepted May 18, 2010.

Disclosure: M.A. Contín, None; D.M. Verra, None; G. Salvador, None; M. Ilincheta, None; N.M. Giusto, None; M.E. Guido, None

Corresponding author: Mario E. Guido, CIQUIBIC, Departamento de Química Biológica, Facultad de Ciencias Químicas, Universidad Nacional de Córdoba-CONICET, Ciudad Universitaria, 5000 Córdoba, Argentina; mguido@fcq.unc.edu.ar. of $\mathrm{Ca}^{2+}$. (Invest Ophthalmol Vis Sci. 2010;51:5491-5498) DOI:10.1167/iovs.10-5643

Tn vertebrates, intrinsically photosensitive retinal ganglion 1 cells (ipRGCs) ${ }^{1,2}$ are responsible for transducing information about ambient lighting conditions to brain areas involved in non-image-forming tasks (e.g., entrainment of the circadian clock and pupillary light reflexes). ${ }^{3-6}$ These ipRGCs may have evolved from a common ancestor with invertebrate rhabdomeric photoreceptors and coexist with ciliary photoreceptors (rods and cones) in the vertebrate retina. ${ }^{7-15}$ Melanopsin (Opn4), the major photopigment present in ipRGCs of different vertebrates, ${ }^{7}$ has been implicated in the photosensitivity of ipRGCs ${ }^{2,4,11-13,15,16}$; this photopigment is closely related to the invertebrate Gq-coupled visual pigment. Two quite separate Opn 4 genes have evolved in vertebrates: Opn $4 m$ and Opn $4 x$, which are mammalian and nonmammalian vertebrate orthologs, respectively. ${ }^{17-20}$ In the chicken, the expression of Opn 4 genes has been reported in diverse retinal cell layers, including RGCs of developing and mature retinas of wild-type animals and the inner retina of blind birds. ${ }^{6,17-23}$ These two genes encode at least five isoforms, ${ }^{17-20}$ two of which show blue light sensitivity. ${ }^{18}$ Moreover, other putative nonopsin photopigments, or cryptochromes (Crys), have been reported to be expressed in the chicken retina. ${ }^{15,24-27}$

Results of electrophysiological and pharmacologic studies with ipRGCs and melanopsin-expressing cultured cells suggest that the biochemical events of phototransduction involve a canonical phosphoinositide (PIP) cascade similar to that of the invertebrate Gq-coupled visual pigment.9,12,13,15,28 After light stimulation, the photocascade in Drosopbila photoreceptors involves the activation of a phospholipase $\mathrm{C} \beta$ (PLC $\beta)$, causing the hydrolysis of phosphatidylinositol 4,5-bisphosphate $\left(\mathrm{PI}(4,5) \mathrm{P}_{2}\right)$ and generating inositol 1,4,5-trisphosphate $\left(\mathrm{IP}_{3}\right)$ and diacylglycerol (DAG), ${ }^{29,30}$ a process that ultimately activates at least two classes of the large TRP ion channel superfamily. ${ }^{29,31,32}$ In ipRGCs, light activates signaling through Gq/11-class $G$ proteins involving PLC activity, elevation of cytoplasmic $\mathrm{Ca}^{2+}$ levels, and induction of membrane depolarization., 10,12,13,15,16,33,34 In earlier work, we have shown the presence of the Gq mRNA in chicken RGC cultures and that treatment with different PLC inhibitors abolishes the light-suppressive effect on ${ }^{3} \mathrm{H}$-melatonin synthesis. ${ }^{15}$ Subsequently, in whole-cell recordings of dissociated mouse retinal cell cultures, specific blockers of PLC and Gq/11 class $G$ proteins abolish the light responses. ${ }^{34}$ Nevertheless, since the complete phototransduction mechanism taking place in these cells is still not known, it remains unclear whether $\mathrm{PI}(4,5) \mathrm{P}_{2}$ and DAG are associated with the light responses of ipRGCs. Moreover, there is no direct report in the literature on the activation of PIP enzyme activities in any vertebrate cell in response to light, whereas work in Drosophila rhabdomeric phototransduction supports PIP involvement by using mutants 
of the PIP cycle enzymes. ${ }^{35,36}$ In the current work, we evaluated light-induced activation of PLC activity, of the PIP cycle enzymes, and of changes in intracellular $\mathrm{Ca}^{2+}$ responses in chicken RGCs. We used immunopurified RGC cultures from embryonic day (E)8 chickens exposed to light or maintained in the dark and evaluated the formation of the PLC activity product $\mathrm{IP}_{3}$; the mobilization of intracellular $\mathrm{Ca}^{2+}$; and the enzyme activities of the PIP cycle, such as DAG kinase (DAGK), phosphatidylinositol kinase (PIK), and phosphatidylinositol phosphate kinase (PIPK).

\section{Materials ANd Methods}

All reagents were analytical grade. $\alpha$-Tubulin ( $\alpha$-Tub) was detected by the mouse monoclonal DM1A antibody (Sigma-Aldrich, St. Louis, MO). The secondary antibodies were AlexaFluor 488 goat anti-rabbit, AlexaFluor 488 goat anti-rat IgG, and AlexaFluor 546 goat anti-mouse IgG (Invitrogen-Molecular Probes, Eugene, OR). Aqueous mounting medium (FluorSave) was from Calbiochem (San Diego, CA). Propidium iodide, protease inhibitor, PLC inhibitor, and other biochemical reagents were from Sigma-Aldrich;-pluronic acid F-127 and Fluo-3 AM were from Invitrogen-Molecular Probes; and serum free supplement (B-27 Supplement 50X) was from Invitrogen-Gibco (Grand Island, NY). The primary antibody against the chicken Opn $4 \mathrm{x}$ was raised in rabbit with the specific melanopsin $\mathrm{x}$ peptide $1 \mathrm{RQKRDLLPDSYSCSEE},{ }^{23}$ the kind gift of Martin Zatz and Arjun Natesan (NIH, Bethesda, MD) and of Ignacio Provencio and Ana M. Castrucci (University of Virginia Charlottesville, VA); the anti-chicken Opn $4 \mathrm{~m}$ was raised in rat and generated with the specific Opn $4 \mathrm{~m}$ peptide: CKHGNRELQKQYHR (BioSynthesis Inc., Lewisville, TX). This antiserum recognizes only the chicken Opn $4 \mathrm{~m}$ and was tested by immunochemistry with positive immunostaining in chicken RGCs of the developing and mature retinas and in primary RGC cultures. No staining was visualized in control cells (CHO cells and primary cultures of rat hippocampal neurons) (Verra DM, unpublished data, 2010).

All experiments were performed in accordance with the ARVO Statement for the Use of Animals in Ophthalmic and Vision Research and were approved by the local animal care committee (School of Chemistry, Universidad Nacional de Córdoba-Córdoba, Exp. 15-9939796).

\section{Chicken Thy-1, Antisera Preparation, and Purification}

Preparation of anti-chicken Thy-1 sera was performed by Bio-Synthesis, Inc., with the NH2-KNITVIKDKLEKC-OH peptide sequence conjugated with KLH to immunize two rabbits. After 6 weeks, serum was obtained from the rabbits and tested by ELISA. A total of 100 to $150 \mathrm{~mL}$ of crude serum with five boosts and four bleeds was purified by affinity column purification.

\section{Primary Cultures of Embryonic RGCs}

RGCs were purified from E8 neural retinas dissected in ice-cold $\mathrm{Ca}^{2+}$. $\mathrm{Mg}^{2+}$-free Tyrode's buffer containing $25 \mathrm{mM}$ glucose, as described elsewhere,${ }^{37}$ with modifications ${ }^{38}$ Briefly, after trypsin dissociation, the cell suspension from 30 to 60 retinas was poured into Petri dishes pretreated with $2.5 \mu \mathrm{g} / \mathrm{mL}$ protein $\mathrm{A}$, followed by incubation at $37^{\circ} \mathrm{C}$ for 30 minutes with an anti-chicken Thy-1 polyclonal antibody (pAb). After the cells were washed exhaustively, identical aliquots of the remaining bound RGCs were harvested in Dulbecco's modified Eagle's medium (DMEM) containing serum free supplement (B27; dilution: 1:500 vol/vol; Invitrogen-Gibco) and seeded in Petri dishes previously treated with $10 \mu \mathrm{g} / \mathrm{mL}$ polylysine and $5 \mu \mathrm{g} / \mathrm{mL}$ laminin; some of with 12-mm glass coverslips. The RGC cultures were incubated at $37^{\circ} \mathrm{C}$ under constant $5 \% \mathrm{CO}_{2}$-air flow in a humid atmosphere. All control cultures were maintained in complete darkness, with no ambient light exposure at any time.

\section{Immunochemistry}

Tissue was treated according to Bobu et al. ${ }^{39}$ Eyes were fixed overnight in $4 \%$ paraformaldehyde at $4{ }^{\circ} \mathrm{C}$, transferred to an ascending series of sucrose solutions $(10 \%, 20 \%$, and $30 \%$, each for 2 hours), and embedded (Tissue-Tek; Sakura Fintek, Tokyo, Japan); 12- $\mu$ m-thick cryostat sections were prepared and stored at $-20^{\circ} \mathrm{C}$ until ready for use. The sections were permeabilized with Triton X-100 (0.1\% in PBS for 5 minutes) and then saturated with PBS containing 0.1\% BSA, $0.1 \%$ Tween-20, and $0.1 \%$ sodium azide (buffer D) for 1 hour. Retinal sections and RGC cultures were fixed for 30 minutes (4\% paraformaldehyde in phosphate-buffered saline; PBS), washed in PBS, and incubated with monoclonal anti $\alpha$-tubulin (DM1A dilution 1:1000; Sigma) at $4^{\circ} \mathrm{C}$ for 24 hours, anti-chicken Opn $4 x$ (dilution. 1:2000) at $4^{\circ} \mathrm{C}$ for 48 hours, or anti-chicken Opn $4 \mathrm{~m}$ (dilution 1:500) at $4^{\circ} \mathrm{C}$ for 48 hours. They were then rinsed in PBS and incubated with AlexaFluor 488 goat anti-rabbit, AlexaFluor 488 goat anti-rat IgG, or AlexaFluor 546 goat anti-mouse IgG (dilution 1:1000) for 1 hour at room temperature. In some experiments, the samples were incubated with propidium iodide $(0.05 \mathrm{mg} / \mathrm{mL})$ and visualized by confocal microscopy (FV1000; Olympus, Lake Success, NY).

\section{$\mathrm{IP}_{3}$ Assessment}

RGC cultures were metabolically labeled with $2 \mu \mathrm{Ci} \cdot \mathrm{mL}^{-1}$ of myo$\left[2{ }^{3} \mathrm{H}(\mathrm{N})\right]$ inositol (PerkinElmer-Life and Analytical Sciences, Waltham, MA) during 48 hours. The cells were then stimulated with cool, white fluorescent light (1200 lux) during different times, according to conditions used to depolarize the ipRGCs in mammals, ${ }^{16}$ in the presence of $10 \mathrm{mM} \mathrm{LiCl}$. The lipids were recovered by TCA extraction meth$\mathrm{ods}^{40-42}$ and the IPs were recovered from the protein/membrane pellets. ${ }^{43}$ The IPs were then separated on resin columns (Dowex AG1-X8; Bio-Rad Laboratories, Hercules, CA) and eluted with increasing concentrations of ammonium formate and formic acid, according to published methods. ${ }^{40,41}$ The IP content was determined in a scintillation counter.

\section{PIP Labeling of RCG Cultures}

After experimental treatment, PIP kinase activities (PIK and PIPK) were assayed by adding $100 \mu \mathrm{L}$ of $2 \times$ assay buffer (500 $\mu \mathrm{M}$ ATP with $4 \mu \mathrm{Ci}\left[\gamma^{-32} \mathrm{P}\right] \mathrm{ATP}, 10 \mathrm{mM} \mathrm{MgCl}_{2}$, and $20 \mathrm{mM}$ HEPES buffer [pH 7.5]) to $100 \mu \mathrm{L}$ of cellular lysates $(200 \mu \mathrm{g}$ protein). The reaction was performed for 5 minutes at $37^{\circ} \mathrm{C}$ and stopped by the addition of $5 \mathrm{~mL}$ of chloroform/methanol (2:1, vol/vol). Blanks were prepared identically, with membranes boiled for 5 minutes before use. Lipid extraction and separation were performed as published, ${ }^{44}$ with slight modification. The lipids were separated by thin-layer chromatography (TLC) on oxalate-coated plates (silica gel 60), with a solvent of chloroform/ methanol/acetone/glacial acetic acid/water (40:13:15:12:7.5, vol/vol). After development, the lipids were visualized with iodine vapor. The spots were scraped from the plate and counted for radioactivity by liquid scintillation counting. PIPK products were identified on the basis of their relative mobility on TLC plates versus commercial standards.

\section{DAGK Activity in RGC Lysates}

DAGK activity was also determined in the RGC lysates by measuring radioactive phosphate incorporation into phosphatidic acid (PA) using $\left[{ }^{32} \mathrm{P}\right] \mathrm{ATP}$ and endogenous DAG as substrates. The standard assay contained $50 \mathrm{mM}$ HEPES (pH 7.4), $20 \mathrm{mM} \mathrm{NaF}, 10 \mathrm{mM} \mathrm{MgCl}_{2}, 1 \mathrm{mM}$ DTT, and $500 \mu \mathrm{M}$ ATP in a volume of $200 \mu \mathrm{L}$. When a micelle-based assay system was used, sodium deoxycholate $(1 \mathrm{mM})$, Triton X-100 (15 mM), or octyl- $\beta$-glucopyranoside $(50 \mathrm{mM})$ was used as a detergent. The reactions were performed at $37^{\circ} \mathrm{C}$ for 5 minutes and stopped by adding chloroform/methanol/1 $\mathrm{N} \mathrm{HCl} \mathrm{(2:1:0.2} \mathrm{by} \mathrm{volume),} \mathrm{after} \mathrm{which} \mathrm{the}$ lipids were extracted. ${ }^{45}$ Lipid extracts were washed five times with theoretical upper phase to eliminate $\left[{ }^{32} \mathrm{P}\right] \mathrm{ATP}$. PA was separated by TLC on $1 \%$ potassium oxalate in silica gel $\mathrm{H}$ and developed with 
chloroform/acetone/methanol/acetic acid/ water (40:15:13:12:7.5, by vol). Lipids visualized by exposure of the chromatograms to iodine vapors were scraped into vial for counting by liquid scintillation.

\section{Calcium Imaging by Fluo-3 AM Fluorescence Microscopy}

Cells were grown in an eight-well recording chamber (Laboratory-Tek; Nunc, Rochester, NY) to $40 \%$ to $50 \%$ confluence and then incubated in colorless DMEM (Invitrogen-Gibco) containing $0.1 \%$ of pluronic acid F-127 and $5 \mu \mathrm{M}$ Fluo-3 AM (Invitrogen-Molecular Probes), a $\mathrm{Ca}^{2+}$ indicator dye, for 30 minutes at $25^{\circ} \mathrm{C}$. The fluorescence imaging technique was performed as published, ${ }^{33,46}$ with modifications by exciting the Fluo-3 AM at $515 \mathrm{~nm}(25.8 \mu \mathrm{W}$ laser intensity) with a laser coupled to a confocal microscope (FluoView-300; Olympus). The emitted fluorescence was captured every 2 seconds, with an oil-immersion objective (PlanApo N 60×Uplan SApo, NA 1.42; Olympus). The 12-bit, $4 \times 4$ binned fluorescence images for each micrograph were used to quantify the fluorescence levels in the cells (MetaMorph, ver. 4.5; Universal Imaging, Downingtown, PA). The mean fluorescence intensity in each cell was background-corrected by subtracting the mean fluorescence of an area with no cells. The mean intensity over a particular area of cells in a selected field was measured in each captured image series. Changes in fluorescence levels were quantified as the ratio between each relative intensity level measured after a light stimulus of 1200 lux $(F)$ and the mean of intensities of 50 serial micrographs before stimulation $\left(F_{0}\right)$. Values of $F / F_{0}$ are not linearly related to changes in $\left[\mathrm{Ca}^{2+}\right]_{\mathrm{i}}$ but are intended to provide a qualitative indication of variations in $\left[\mathrm{Ca}^{2+}\right]_{\mathrm{i}}$. No significant vehicle effects or changes in focus were detected. Responses were considered significant when the ratio at the peak differed from the baseline levels by at least $20 \%$.

RNA Isolation and cDNA Synthesis. Total RNA was extracted from RGC cultures according to a published method ${ }^{47}$ (TRIzol; Invitrogen) and treated with DNase (Promega, Madison, WI). cDNA was synthesized with M-MLV (Promega) by using oligo(dT). Each RT-PCR quantitation experiment was performed in triplicate in three independent experiments.

PCR Primers. The following Gallus gallus sequences were used: glyceraldehyde 3-phosphate dehydrogenase (GAPDH; accession K01458), Opn4x (accession AY036061), Opn $4 m$ (accession NM_204625), Gallus gallus red cone-opsin (accession NM_205440), cryptochrome 1 (Cry1; accession NM_204245), and cryptochrome 2 (Cry2; accession NM_204244). Oligonucleotides were designed by using a program that generates 200- to 600-base-pair products with minimal hairpin or loop formation (NTI Advance 10; Vector Laboratories, Burlingame, CA). The oligonucleotide sequences used for RT-PCR were as follows: GAPDH forward: 5'-AGGCGAGATGGTGAAAGTCG3', reverse: 5'-TCTGCCCATTTGATGTTGCT-3'; Cry 1 forward: 5'-AGAGAGTGTCCAGAAGGCTGCAAA-3', reverse: 5'-ACTGTTGCAAGAAGACCCAGTCCT-3'; Cry2 forward: 5'-GCCAAGTGCATCATTGGAGTGG3', reverse: 5'-CTTCAGTGCACAGCTCTTCTGCTC-3'; Opn $4 m$ forward: 5'-TCTCGCCGTAGAACATCC-3', reverse: 5'-GAAGTGTTTCAGAGCAAGGTAGGA-3'; Op $44 x$ forward: 5'-TGTAGAGCTTGACACTGTAGAACCA-3', reverse: 5'-TGACCGCCCTAGCACCTT-3'; and red cone opsin forward: 5'-AAGGCCAAGACTTCTACGTGC3', reverse: 5'-CGAGATCTGGTTGATGACGCT- $3^{\prime}$.

\section{Statistics}

Statistics involved one- or two-way analysis of variance (ANOVA) with the Duncan post hoc test or Student's $t$-test, as appropriate (significance at $P<0.05$ ).

\section{Results}

\section{Characterization of Primary Cultures of RGCs}

To study the phototransduction mechanisms taking place in ipRGCs, we used primary cultures of immunopurified chicken
RGCs at E8, which have been described as intrinsically photosensitive. ${ }^{15}$ These highly pure cultures of RGCs allow the direct study of light responses in the absence of cone and rod photoreceptors and show the early expression of different potential photopigment mRNAs such as Opn $4 x$, Opn $4 m$, Cry 1 , and Cry2, but not of the red cone opsin (Fig. 1A). Figure 1B showing immunopurified cells by Thy 1 -antibody immunopanning, ${ }^{15,37,38}$ indicates that a percentage $(\geq 20 \%)$ of total cells stained with $\alpha$-tubulin (Fig. 1Bb) or propidium iodide (Fig. 1Be) at E8 and maintained for 3 to 5 days in the cultures, expressed both melanopsin proteins: Opn $4 x$ (Fig. 1Ba; $11 \% \pm 2 \%$ of total cells, $n=1557$ cells) and the Opn $4 \mathrm{~m}$ (Fig. 1Bd; 22\% \pm $2 \%$ of total cells, $n=2500$ cells). Moreover, a developing retinal section from a chick embryo at the same stage (E8) clearly showed positive immunoreactivity for both the Opn $4 x$ and Opn $4 \mathrm{~m}$ proteins, which are mainly localized in the forming layer of RGCs (Figs. 1Bc, 1Bf).

Based on the photopigment complexity observed, we further investigated the biochemical events occurring downstream of the photon capture, in immunopurified RGC cultures.

\section{Changes in Intracellular $\mathrm{Ca}^{2+}$ Levels of RGCs after Light Exposure}

Our previous observations showed that the inhibitory effect of light on melatonin synthesis in chicken ipRGCs was reversed by the administration of the $\mathrm{Ca}^{2+}$ chelator BAPTA-AM $(10 \mathrm{mM})$ or lanthanum $\left(\mathrm{La}^{3+} ; 1 \mathrm{mM}\right)$, a known TRPC/TRPL-channel blocker. ${ }^{15}$ To further confirm the direct participation of $\mathrm{Ca}^{2+}$ in the photic responses of chicken ipRGCs, we measured $\mathrm{Ca}^{2+}$ levels by using Fluo-3 AM confocal microscopy of individual cultured RGCs, before and after 30 seconds of bright light stimulation (Fig. 2, arrows). The results indicated that $\sim 20 \%$ of the cells in each culture displayed a significant increase in somatic $\mathrm{Ca}^{2+}$ levels after light stimulation (black line) with differential responses as illustrated for individual cells (Figs. 2A, 2B, 2D, white arrowheads), whereas more than $75 \%$ of the cells did not respond to the photic input (Figs. 2A, 2B, gray line; 2D, white arrows).

Most striking, we observed different types of photic responses by $\mathrm{Ca}^{2+}$ imaging in the cell population tested (Fig. 2C), which can be classified as sustained (Fig. 2A, black line) and transient (Fig. 2B, black line) responses. Sustained responses were at an amplitude of $\sim 50 \%$ with respect to basal levels and a time to peak of $\sim 250$ seconds. By contrast, transient responses exhibit an amplitude of $\sim 30 \%$ with a time to peak of $\leq 30$ seconds. Moreover, when the PLC inhibitor U73122 (5 $\mu \mathrm{M})$ was applied to the cultures before light stimulation, no $\mathrm{Ca}^{2+}$ variations were seen in any of the tested cells (data not shown).

\section{IP $_{3}$ Production by Light}

Cultures previously incubated with myo- ${ }^{3} \mathrm{H}$ inositol for 48 hours were light-stimulated during 90 seconds, to further investigate PLC activity directly in RGCs. $\mathrm{IP}_{3}$ production was then evaluated as a major product of PLC activity in bright light and in control cells maintained in the dark. We found a significant increase $(30 \%)$ in levels of labeled $\mathrm{IP}_{3}$ in cultures treated with $10 \mathrm{mM} \mathrm{LiCl}$, the well-known inositol monophosphate phosphatase (IPP) inhibitor, and exposed to light compared with the control cells kept in the dark (Fig. 3A; $P<0.04$ ). Moreover, when we assessed the effect of the specific PLC inhibitor $(5 \mu \mathrm{M})$ on the cultures, we found no increase in levels of radiolabeled $\mathrm{IP}_{3}$ after light stimulation, compared with the dark condition or vehicle-treated cultures exposed to light (Fig. $3 \mathrm{~A})$. The rapid activation of PLC by light caused a fast hydrolysis of $\mathrm{PI}(4,5) \mathrm{P}_{2}$ and other PIPs, together with the concomi- 
A
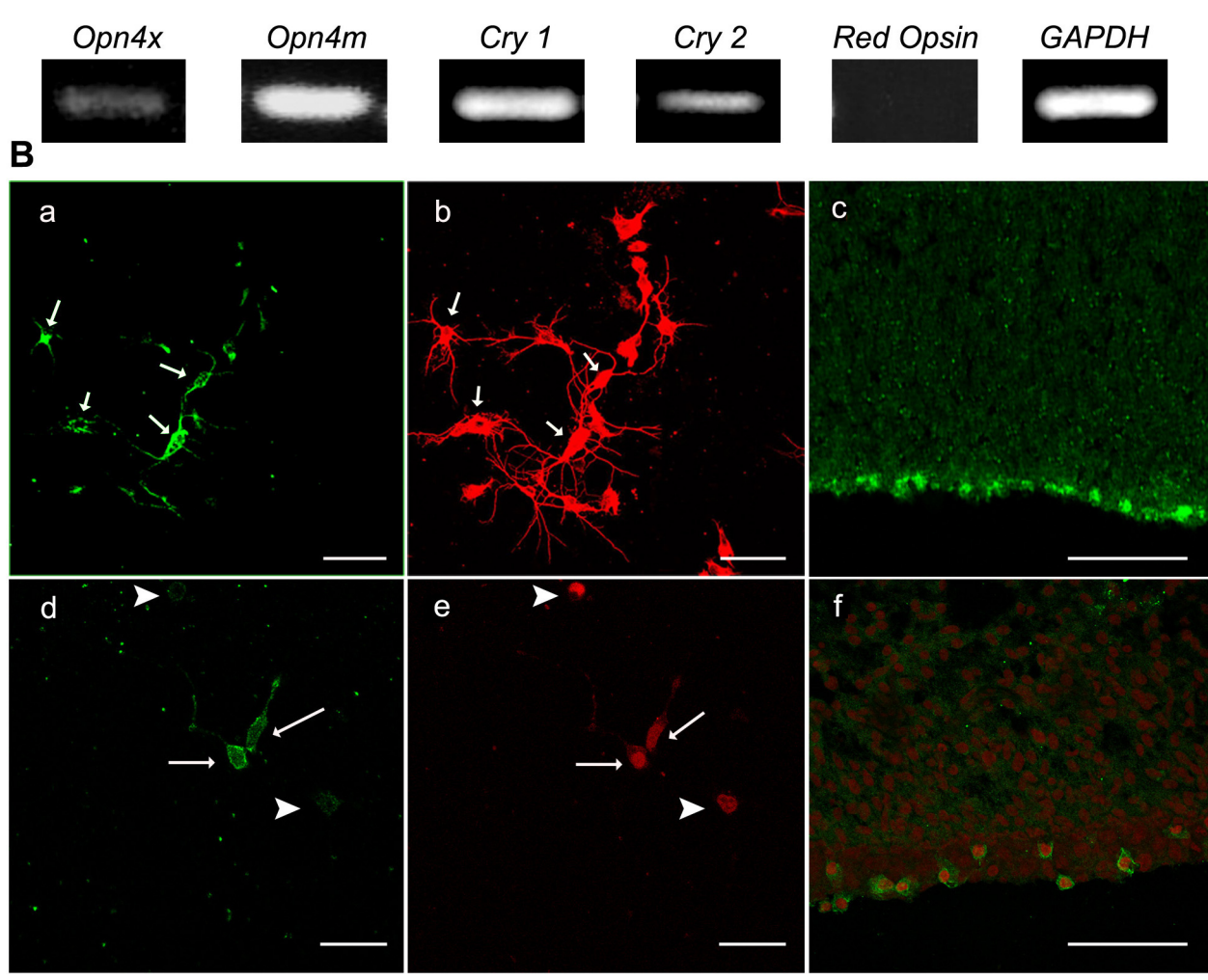

FigURE 1. Expression of different nonvisual photopigments in immunopurified RGC cultures and retinal sections from E8 chicks. (A) RT-PCR of RGC cultures showed detectable mRNA levels of Opn $4 x$, Opn $4 m, C r y 1$, and Cry 2 but not of red cone opsin. (B) Primary cultures of embryonic RGCs maintained for 3 to 5 days were immunolabeled for $\alpha$-tubulin (Bb) or propidium iodide (Be) and Opn4x-like (Ba) or Opn4m-like (Bd) proteins with specific primary antibodies and visualized by confocal microscopy. Arrows: Opn4 (+) cells located both in a few cells in the primary cultures of RGCs (Ba, Bd) and the RGC layer (Bc, Bf); arrowbeads: cells nonimmunoreactive for Opn $4 \mathrm{~m}$ (Bd, Be). Chick embryonic retinal sections dissected at E8 were immunolabeled for Opn $4 x$-like (Bc) or Opn $4 \mathrm{~m}$-like (Bf) protein and propidium iodide and visualized by confocal microscopy with positive immunostaining located in the GCL of the developing retina. Scale bar: (Ba, Bb, Bd, Be) $10 \mu \mathrm{m}$; (Bc, Bf) $40 \mu \mathrm{m}$. tant and transient formation of $\mathrm{IP}_{3}$ and other inositol phosphates. A complication in monitoring changes in PIPs alone is the cells' ability to resynthesize phosphatidylinositol (PI) rap- idly, and therefore also $\mathrm{PIP}$ and $\operatorname{PI}(4,5) \mathrm{P}_{2}$. For this reason, we inhibited IPP with $\mathrm{LiCl}(10 \mathrm{mM})$, thus allowing the accumulation of $\mathrm{IP}_{3}$ products over a longer stimulation time. ${ }^{48}$ To see

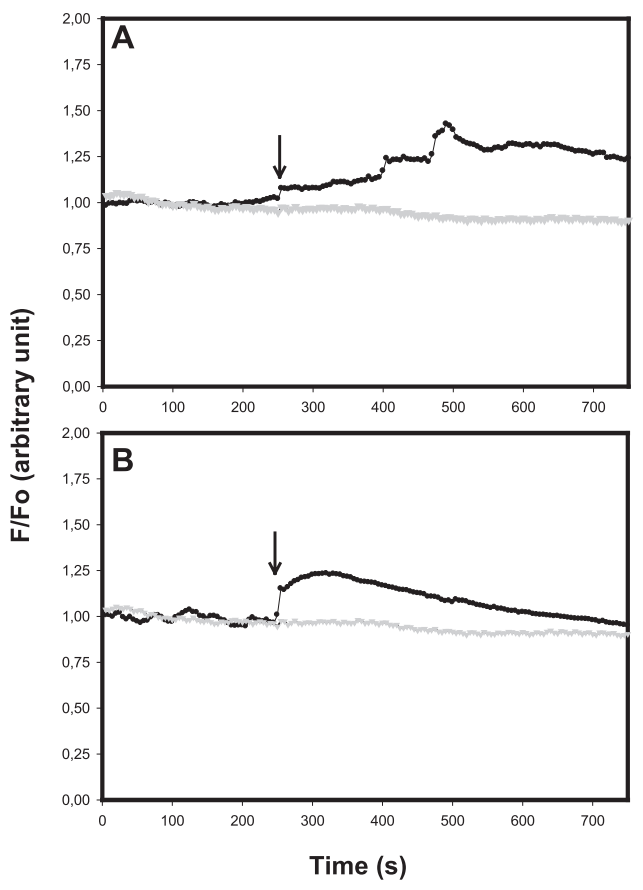

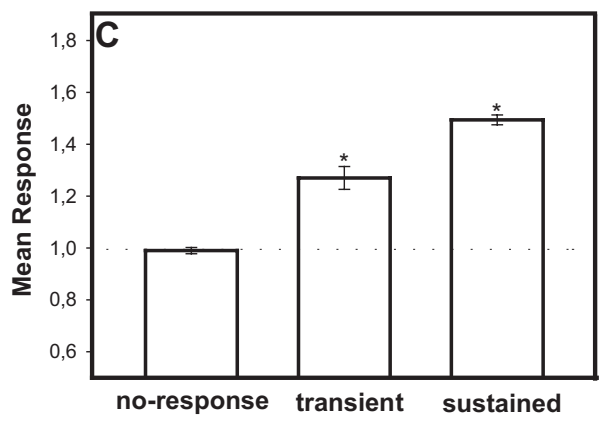

D

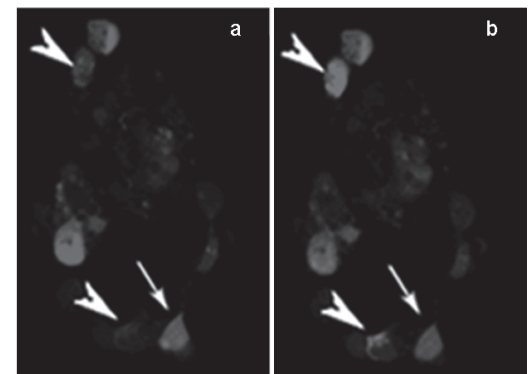

FIGURE 2. Light effect on $\left[\mathrm{Ca}^{2+}\right]_{\mathrm{i}}$ levels in RGC cultures. RGC cultures were maintained in constant darkness. On day 5, the cultures were loaded with Fluo-3 AM and stimulated with bright white light for 30 seconds. (A, B) Graphic representations showing the light's effect on differential $\mathrm{Ca}^{2+}$ responses by fluorescence imaging in individual cultured RGCs. (A, B) Sustained (A, black line), transient (B, black line), and no (A, B, gray line $) \mathrm{Ca}^{2+}$ responses after light stimulation. Changes in fluorescence levels were quantified as the ratio between each relative intensity level measured after a light stimulus of 1200 lux $(F)$ and the mean of intensities in 50 serial images before stimulation $\left(F_{0}\right)$. Values of $F / F_{0}$ were not linearly related to changes in $\left[\mathrm{Ca}^{2+}\right]_{\mathrm{i}}$ but provided a qualitative indication of variations in $\left[\mathrm{Ca}^{2+}\right]_{\mathrm{i}}(n=$ 125 cells tested). Sustained and transient responses present an amplitude $\left(\Delta F / F_{0}\right)$ of $1.51 \pm 0.02$ and $1.27 \pm$ 0.04 , respectively, over the arbitrary value of 1.00 assigned to nonresponsive cells $(n=11-13$ from three independent experiments) and a time to peak of 250 and 30 seconds, respectively. Data are expressed as the mean \pm SD. Sustained responses $(P \leq 0.0001 ; t$-value 9.7$)$ and transient responses $\left(P \leq 0.001, t\right.$-value 11.6) compared with the responses of the nonphotosensitive cells $\left(\Delta F / F_{0}=1.08 \pm 0.09\right)$ by Student's $t$-test. (C) Mean normalized results of the photic effect on the fluorescence increased over basal levels (no response) in RGC cultures, showing transient and sustained light-evoked responses. Histograms represent the mean \pm SE $\left(n=11-15\right.$ for each group). ${ }^{*} \leq \leq 0.001$. (D) Fluo-3 AM imaging showing the fluorescence before (Da) and after (Db) light stimulation by confocal microscopy. Arrowheads: detectable variations in fluorescence levels; arrows: no significant fluorescence changes after photic stimulation. Scale bar, $40 \mu \mathrm{m}$. 


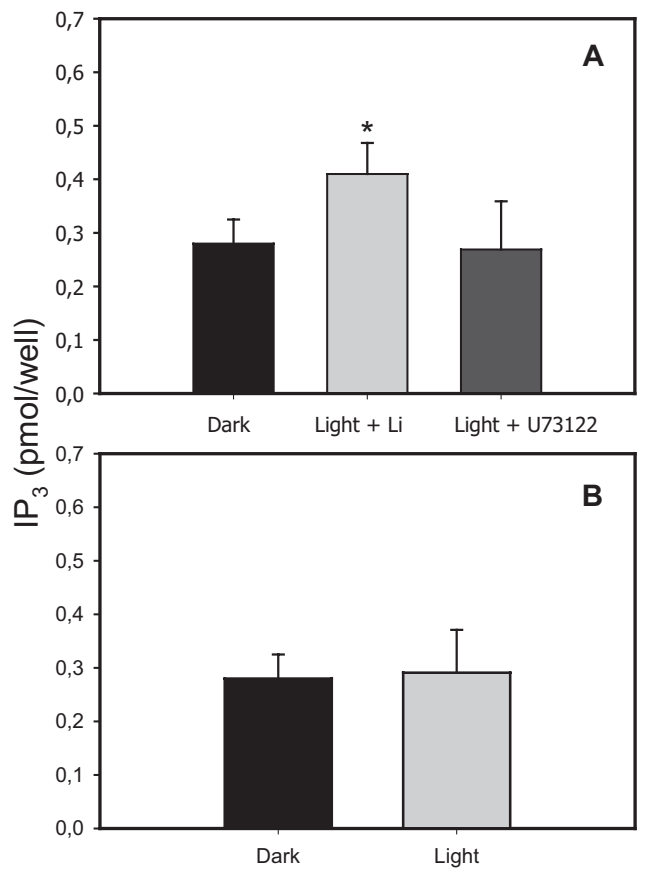

FiguRE 3. Effects of light on formation of ${ }^{3} \mathrm{H}-\mathrm{IP}{ }_{3}$ in RGC cultures. RGC cultures were fed with $2 \mu \mathrm{Ci} \cdot \mathrm{mL}^{-1}$ myo- $\left[2-{ }^{3} \mathrm{H}(\mathrm{N})\right]$ inositol for 48 hours in the dark and assayed for formation of $\left[{ }^{3} \mathrm{H}\right]$-inositol phosphates. Data are expressed as the mean $\pm \operatorname{SEM}(n=3-4$ /group). The data shown are representative of observations in three independent experiments. (A) Effect of light on ${ }^{3} \mathrm{H}-\mathrm{IP}_{3}$ levels in the presence of $10 \mathrm{mM} \mathrm{LiCl}(\mathrm{Li}$, light gray bar) or $10 \mathrm{mM} \mathrm{Li}+5 \mu \mathrm{M} \mathrm{U} 73122$, the PLC inhibitor (dark gray bar). ${ }^{*} P<0.04$ compared with the dark control (black bar). (B) Effect of the light on ${ }^{3} \mathrm{H}-\mathrm{IP}_{3}$ levels in the absence of $10 \mathrm{mM} \mathrm{Li}$ inhibitor (light gray bar) and in the dark control (black bar).

whether IP products produced by light were metabolized after light stimulation, we performed a series of experiments in the absence of $\mathrm{LiCl}(10 \mathrm{mM})$. No light/dark differences were observed (Fig. 3B), indicating the rapid cellular metabolism of IPs.

The phototransduction cascade is one of the fastest known $G$ protein-coupled signaling cascades. ${ }^{29,31}$ Thus, we speculate

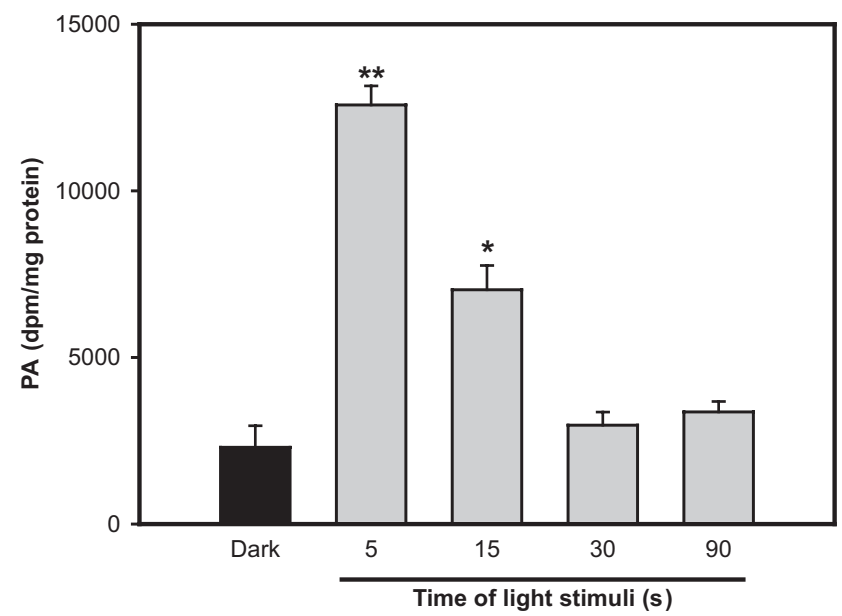

FIGURE 4. Light effect on DAGK activity in RGC cultures. The cultures were maintained in the dark, and, on day 5 , the cells were stimulated with bright white light for $5,15,30$, or 90 seconds, whereas the control cells remained in the dark. The cells were then collected to measure DAGK activity. Data are expressed as the mean \pm SEM $(n=$ 3-4/group) from three independent experiments. ${ }^{*} P<0.05,{ }^{* *} P<$ 0.007 compared with basal levels of activity in the dark control. that the PLC activity in RGC phototransduction may be a rapid gating mechanism. To evaluate this hypothesis, we assessed PLC activity during the shortest possible stimulation period ranging from 5 to 90 seconds of light exposure by measuring $\mathrm{IP}_{3}$ production after chromatography separation. The results showed higher production of $\mathrm{IP}_{3}$, even after only 5 to 30 seconds of light-induced stimulation, indicating that the $30 \%$ increment observed is generated during the first second of stimulation (data not shown).

\section{DAGK and PIK/PIPK Activities in RGC Cultures}

The spatial restriction and steady state levels of specific PIPs are determined primarily by the concerted action of PIPKs and phosphatases, with tightly controlled localizations. $\mathrm{PI}(4,5) \mathrm{P}_{2}$ is produced locally at the plasma membrane either by the phosphorylation of PI(4)P (by type I PIPKs) or PI(5)P (by type II PIPKs). ${ }^{49,50}$ DAGK is the enzyme that converts DAG to PA, which is used to resynthesize $\operatorname{PI}(4,5) \mathrm{P}_{2}$.

We investigated DAGK activity in light-stimulated cultures of chicken RGCs and controls maintained in the dark. Cells were light-stimulated during different times and enzyme activity was determined in RGC homogenates by assessing the
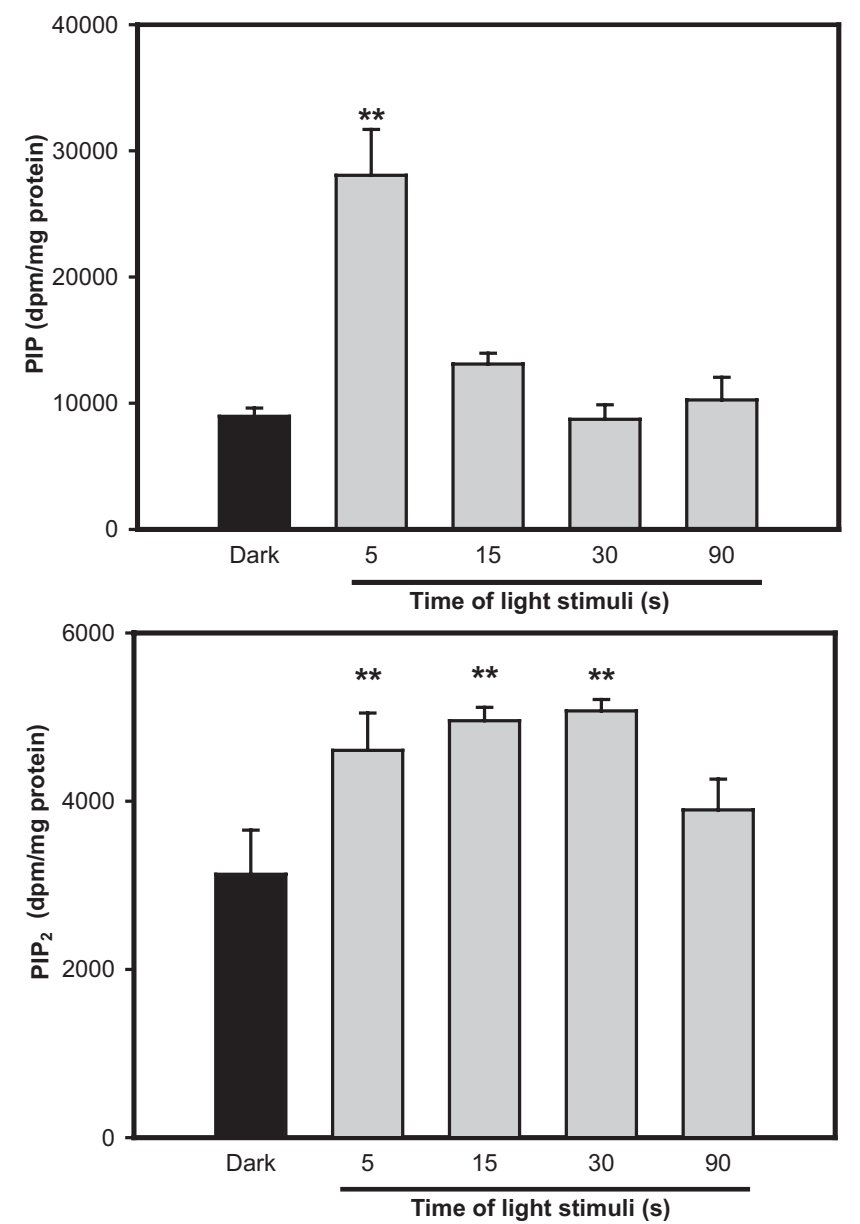

FIGURE 5. Light effect on PI and PIPK activities in RGC cultures. RGC cultures were maintained in the dark and, on day 5 , the cells were stimulated with bright white light for $5,15,30$, or 90 seconds, whereas the control cells remained in the dark. The cells were then collected to assess PIK and PIPK activities. Data are expressed as the mean \pm SEM ( $n=3$-4/group) from three independent experiments. Top: light's effect on PIK activity. ${ }^{* *} P<0.007$ compared with the dark control. Bottom: light's effect on PIPK activity ${ }^{* *} P<0.007$ compared with the dark control. 
incorporation of radioactive phosphate into PA using $\left[{ }^{32} \mathrm{P}\right] \mathrm{ATP}$ and endogenous DAG. As shown in Figure 4, we found a significant increase in $\left[{ }^{32} \mathrm{P}\right] \mathrm{PA}$ formation after only 5 seconds of light stimulation, and the level remained elevated at least up to 15 seconds of light exposure. When DAGK was assayed in RGC cultures (dark and 10 seconds of light) in a micelle-based assay with $\mathrm{Na}$ deoxycholate as the detergent, the $\left[{ }^{32} \mathrm{P}\right] \mathrm{PA}$ levels were $138 \%$ higher than in the standard assay without detergent, and a similar light-stimulated activity pattern was observed (data not shown). However, when $10 \mathrm{mM}$ Triton X-100 was present, $\left[{ }^{32} \mathrm{P}\right] \mathrm{PA}$ formation was strongly inhibited (data not shown).

To study the effect of light on $\mathrm{PI}(4,5) \mathrm{P}_{2}$ levels, we assayed PI and PIPK activities in chick RGC cultures exposed to light for different lengths of time (5-90 seconds) or maintained in the dark. The kinase activities were determined in the cellular homogenates and evaluated by assessing the incorporation of ${ }^{32} \mathrm{P}$ from $\left[{ }^{32} \mathrm{P}\right] \mathrm{ATP}$ into PIP or $\mathrm{PI}(4,5) \mathrm{P}_{2}$ in the presence of the endogenous substrates.

The results depicted in Figure 5 show a significant increase in $\left[{ }^{32} \mathrm{P}\right]$ PIP labeling at 5 seconds of light stimulation, indicating that PIK was very rapidly and transiently activated during a brief light pulse. In addition, $\left[{ }^{32} \mathrm{P}\right] \mathrm{PI}(4,5) \mathrm{P}_{2}$ levels peaked after 5 seconds of light stimulation. The increased $\left[{ }^{32} \mathrm{P}\right] \mathrm{PI}(4,5) \mathrm{P}_{2}$ levels were maintained during up to 30 seconds of exposure to light, unlike in the dark control.

\section{Discussion}

In this work, primary cultures of immunopurified chicken RGCs at very early embryonic stages (E8) responded to light through a photoactivated biochemical cascade involving the activation of the enzyme PLC with a concomitant increase in the level of $\mathrm{IP}_{3}$, activation of the PIP cycle, and mobilization of intracellular $\mathrm{Ca}^{2+}$. This is the first study to directly relate the phototransduction cascade and the PIP cycle in ipRGCs of the vertebrate retina.

In a previous study, we showed that the phototransduction cascade in cultures of chicken RGCs could activate a PLC enzyme effector. ${ }^{15}$ These results are in agreement with previous data reported in Xenopus melanophores, ${ }^{9,12}$ showing a phosphoinositide signaling pathway similar to that in invertebrate phototransduction. The vertebrate ipRGCs form part of a complex retinal structure with different layers and cellular types. We used Thy-1 immunopurified embryonic chicken RGCs ${ }^{15,37,38}$ that were free of other retinal cell types and at a very early developmental stage at which only RGCs are postmitotic and mostly mature. ${ }^{51}$ As shown in Figure 1, RGCs in primary cultures and in developing retina at the same stage (E8) exhibited expression of the Opn $4 x$ and Opn $4 \mathrm{~m}$ photopigments. Furthermore, after 5 days, primary cultures contained $\sim 20 \%$ of cells displaying positive immunoreactivity for Opn4 photopigments, as well as the expression of other putative photopigments (Cry1 and Cry2; Fig. 1), indicating that a considerable number of Thy-1-positive cells in RGC cultures may be photosensitive.

One striking characteristic of the photosensitivity in vertebrate ipRGCs is that which is related to changes in intracellular $\mathrm{Ca}^{2+}$ levels after light exposure, ultimately causing cell depolarization. ${ }^{33,52}$ The chicken RGC cultures showed very rapid and distinct increases in $\mathrm{Ca}^{2+}$ in response to a 30-second light stimulus (Fig. 2). Two types of photic responses were observed: One was sustained over at least 8 to 10 minutes after stimulation, and the other was a transient that disappeared or diminished at longer times after stimulation. These distinct $\mathrm{Ca}^{2+}$ responses most likely reflect different subsets of ipRGCs, as previously described in mammals. ${ }^{33,53,54}$ Most notably, responses were abolished in all cells tested after blockade of PLC activation by the specific inhibitor U73122. This result further supports the idea of a PIP cascade operating in the ipRGCs. In addition, the activation of $\mathrm{Ca}^{2+}$-permeable, light-sensitive channels, such as the TRP and the TRPL channels, may cause the $\left[\mathrm{Ca}^{2+}\right]_{i}$ increase since the treatments with a $\mathrm{Ca}^{2+}$ chelator (BAPTA) or TRP channel blockers $\left(\mathrm{La}^{3+}\right)$ were able to reverse the photoresponses observed. ${ }^{14,15,34}$ However, $\mathrm{IP}_{3}$ receptors triggering a $\mathrm{Ca}^{2+}$ release from intracellular stores may play a modulatory role, but are apparently not essential for ipRGC phototransduction. ${ }^{34}$

In this work, we directly investigated the light activation of PLC activity in RGC cultures by assessing one of its enzymatic products, ${ }^{3} \mathrm{H}-\mathrm{IP}_{3}$, after metabolic labeling. Our findings showed a significant increase $(30 \%)$ in labeled $\mathrm{IP}_{3}$ in cultures exposed to bright white light pulses of 90 seconds, when compared with control cultures kept in the dark (Fig. 3A). This effect was totally suppressed when the cells were treated with the specific PLC inhibitor U73122 (5 $\mu \mathrm{M})$ before light stimulation.

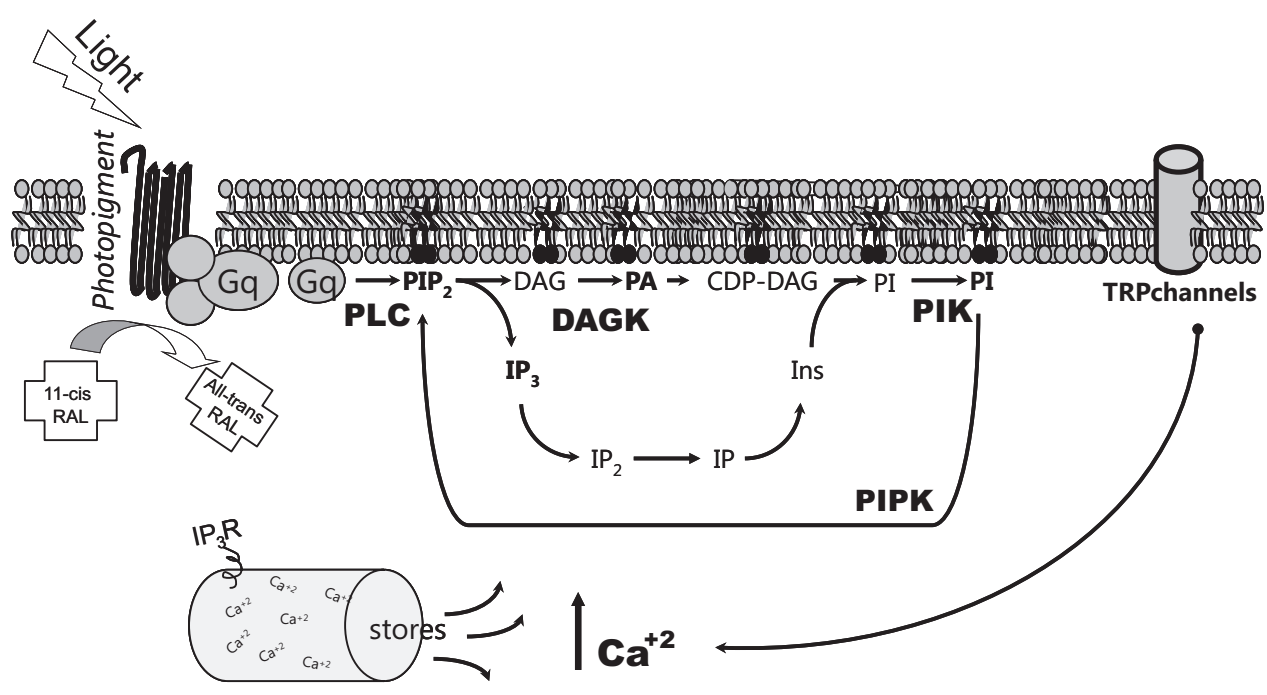

SCHEME 1. The photocascade operating in ipRGCs of the chicken begins when light activates a photopigment such as Opn4, which uses a vitamin A derivative. ${ }^{15}$ Thus, light activation may cause the photoisomerization of 11-cis retinal to all-trans retinal, which may lead to the activation of a $G$ protein (Gq), which in turn activates PLC. PLC hydrolyzes $\mathrm{PI}(4,5) \mathrm{P}_{2}$ to $\mathrm{IP}_{3}$ and DAG. The elevation in levels of $\mathrm{IP}_{3}$ by light is inhibited by $\mathrm{U} 73122(5 \mu \mathrm{M})$, a specific PLC inhibitor, and is visualized after $\mathrm{LiCl}$ pretreatment to avoid rapid metabolism. The DAG is rapidly transformed into PA (during the first 5-10 seconds of light stimulation) by activation of DAGK activity. PA is in turn converted into $\mathrm{PI}(4,5) \mathrm{P}_{2}$ through a multistep process that involves the concerted action of CDP-diacylglycerol synthase (CDS), PI synthase

(PIS), and the two kinases PIK and PIPK. PIK and PIPK activities increase during the first seconds of stimulation. As a consequence of the light activation, there is also a significant increase in intracellular $\mathrm{Ca}^{2+}$ levels, which ultimately contributes cell depolarization, together with the potential activation of TRP-like channels. 
It is highly probable that PLC stimulation occurs within milliseconds of exposure to light, just as it does in the known $G$ protein-coupled signaling cascades. ${ }^{29,31}$ However, this time scale is not compatible with our experimental design. Furthermore, the light-dark differences observed in levels of radiolabeled $\mathrm{IP}_{3}$ in ipRGCs can be both underestimated as a consequence of the longer stimulation times examined and diluted by the IP content in nonphotosensitive RGCs in the cultures.

It is known that the stimulation of PLC activity leads to the diminution of $\mathrm{PI}(4,5) \mathrm{P}_{2}$ levels in favor of the generation of DAG and $\mathrm{IP}_{3}$. DAG is converted into $\mathrm{PA}$ and $\mathrm{PA}$ into $\mathrm{PI}(4,5) \mathrm{P}_{2}$, through a multistep process involving the action of DAGK, CDP-DAG synthase (CDS), phosphatidylinositol synthase (PIS), and the two kinases PIK and PIPK. A complication in monitoring changes in PI alone is the ability of the cells to resynthesize PI rapidly and therefore also PIP and $\mathrm{PI}(4,5) \mathrm{P}_{2} \cdot{ }^{31,55,56}$

We evaluated DAGK, PIK, and PIPK activities in chicken RGC cultures after brief light pulses ranging from 5 to 90 seconds. Our results show a significant activation of DAGK and PIK compared with controls kept in the dark. The quick increase in PA and PIP levels, taking only 5 to 15 seconds to peak, was transient, and the activation disappeared after 10 to 30 seconds of stimulation. In addition, light stimulated PIPK activity after 5 seconds, and $\mathrm{PIP}_{2}$ levels showed a sustained increase after 30 seconds of stimulation. Overall, our findings strongly suggest that PA and PIP production is tightly regulated during the first seconds of light stimulation, most likely to reestablish the steady state concentration of PIP in the membrane, with a very rapid, transient, and sequential activation of lipid kinases. In addition, we have observed that the DAGK present in RGC cultures as assessed in a micelle-based assay system could be a cytosolic (type I) isoform (data not shown). Type I DAGKs $(\alpha, \beta$, and $\gamma)$ have calcium-binding EF hand motifs and are more active in the presence of this ion. ${ }^{57-59} \mathrm{~A}$ DAGK activity strongly inhibited by Triton X-100 was also reported to be present in bovine rod outer segments (ROS). ${ }^{60}$ A DAGK- $\gamma$ was identified in rat and bovine ROS and a lightdependent association was reported. ${ }^{61}$

Other studies suggest that PLC triggers the opening of the light-gated channels by a membrane-associated mechanism, ${ }^{34}$ with the direct interaction between the channels and $\mathrm{PI}(4,5) \mathrm{P}_{2}$ maintaining them in a closed state during darkness. In Drosophila, light stimulates PLC to hydrolyze $\mathrm{PI}(4,5) \mathrm{P}_{2}$, decreasing its concentration and releasing the channels into an open state. ${ }^{32,36}$ Nevertheless, in ipRGCs, it is unclear whether $\mathrm{IP}_{3}$, $\mathrm{PI}(4,5) \mathrm{P}_{2}$, or DAG plays a central role in cell depolarization. We can also speculate that replenishment of $\mathrm{PI}(4,5) \mathrm{P}_{2}$ levels by the sequential activation of PI and PIPKs regulates the concentration of $\mathrm{PI}(4,5) \mathrm{P}_{2}$ to modulate the channel state. These results are in agreement with previous findings in Drosopbila, where disruption of $\mathrm{PI}(4,5) \mathrm{P}_{2}$ regeneration interrupts the phototransduction process. ${ }^{35,62,63}$ Thus, mutations that affect $\mathrm{PI}(4,5) \mathrm{P}_{2}$ regeneration in vertebrates may disrupt phototransduction in the ipRGCs. Mechanisms that promote the regeneration of $\mathrm{PIP}_{2}$ are therefore critical for maintaining signaling capacity.

Overall, our observations further support the idea that the phototransduction cascade operating in primary cultures of chicken RGCs and summarized in Scheme 1 involves a PIPs cascade.

\section{Acknowledgments}

The authors thank Beatriz L. Caputto for critical reading of the manuscript and Carlos Mas, Cecilia Sampedro, Susana Deza, and Gabriela Schachner for excellent technical support.

\section{References}

1. Berson DM, Dunn FA, Takao M. Phototransduction by retinal ganglion cells that set the circadian clock. Science. 2002;295: $1070-1073$.

2. Hattar S, Liao HW, Takao M, Berson DM, Yau KW. Melanopsincontaining retinal ganglion cells: architecture, projections, and intrinsic photosensitivity. Science. 2002;295:1065-1070.

3. Hattar S, Lucas RJ, Mrosovsky N, et al. Melanopsin and rod-cone photoreceptive systems account for all major accessory visual functions in mice. Nature. 2003;424:76-81.

4. Lucas RJ, Hattar S, Takao M, Berson DM, Foster RG, Yau KW. Diminished pupillary light reflex at high irradiances in melanopsinknockout mice. Science. 2003;299:245-247.

5. Panda S, Sato TK, Castrucci AM, et al. Melanopsin (Opn4) requirement for normal light-induced circadian phase shifting. Science. 2002;298:2213-2216.

6. Valdez DJ, Nieto PS, Garbarino-Pico E, et al. A nonmammalian vertebrate model of blindness reveals functional photoreceptors in the inner retina. FASEB J. 2009;23:1186-1195.

7. Provencio I, Rodriguez IR, Jiang G, Hayes WP, Moreira EF, Rollag MD. A novel human opsin in the inner retina. J Neurosci. 2000; 20:600-605.

8. Arendt D. Evolution of eyes and photoreceptor cell types. Int J Devel Biology. 2003;47:563-571

9. Isoldi MC, Rollag MD, Castrucci AM, Provencio I. Rhabdomeric phototransduction initiated by the vertebrate photopigment melanopsin. Proc Natl Acad Sci USA. 2005;102:1217-1221.

10. Koyanagi M, Kubokawa K, Tsukamoto H, Shichida Y, Terakita A. Cephalochordate melanopsin: evolutionary linkage between invertebrate visual cells and vertebrate photosensitive retinal ganglion cells. Curr Biol. 2005;15:1065-1069.

11. Melyan Z, Tarttelin EE, Bellingham J, Lucas RJ, Hankins MW. Addition of human melanopsin renders mammalian cells photoresponsive. Nature. 2005;433:741-745.

12. Panda S, Nayak SK, Campo B, Walker JR, Hogenesch JB, Jegla T. Illumination of the melanopsin signaling pathway. Science. 2005; 307:600-604.

13. Qiu X, Kumbalasiri T, Carlson SM, et al. Induction of photosensitivity by heterologous expression of melanopsin. Nature. 2005; 433:745-749.

14. Warren EJ, Allen CN, Brown RL, Robinson DW. The light-activated signaling pathway in SCN-projecting rat retinal ganglion cells. Eur J Neurosci. 2006;23:2477-2487.

15. Contin MA, Verra DM, Guido ME. An invertebrate-like phototransduction cascade mediates light detection in the chicken retinal ganglion cells. FASEB J. 2006;20:2648-2650.

16. Berson DM. Strange vision: ganglion cells as circadian photoreceptors. Trends Neurosci. 2003;26:314-320.

17. Bellingham J, Chaurasia SS, Melyan Z, et al. Evolution of melanopsin photoreceptors: discovery and characterization of a new melanopsin in nonmammalian vertebrates. PLOS Biol. 2006;4:e254.

18. Torii M, Kojima D, Okano T, et al. Two isoforms of chicken melanopsins show blue light sensitivity. FEBS Lett. 2007;581: 5327-5331.

19. Tomonari S, Takagi A, Akamatsu S, Noji S, Ohuchi H. A noncanonical photopigment, melanopsin, is expressed in the differentiating ganglion, horizontal, and bipolar cells of the chicken retina. Dev Dyn. 2005;234:783-790.

20. Tomonari S, Takagi A, Noji S, Ohuchi H. Expression pattern of the melanopsin-like (cOpn $4 \mathrm{~m})$ and VA opsin-like genes in the developing chicken retina and neural tissues. Gene Expr Patterns. 2007;7:746-753.

21. Bailey MJ, Cassone VM. Melanopsin expression in the chick retina and pineal gland. Brain Res Mol Brain Res. 2005;134:345-348.

22. Chaurasia SS, Rollag MD, Jiang G, et al. Molecular cloning, localization and circadian expression of chicken melanopsin (Opn4): differential regulation of expression in pineal and retinal cell types. J Neurochem. 2005;92:158-170.

23. Lima LH, Scarparo AC, Isoldi MC, Visconti MA, Castrucci AM. Melanopsin in chicken melanocytes and retina. Biol Rhythm Res. 2006;37:393-404. 
24. Bailey MJ, Chong NW, Xiong J, Cassone VM. Chickens' Cry2 molecular analysis of an avian cryptochrome in retinal and pineal photoreceptors. FEBS Lett. 2002;513:169-174.

25. Haque R, Chaurasia SS, Wessel JH 3rd, Iuvone PM. Dual regulation of cryptochrome $1 \mathrm{mRNA}$ expression in chicken retina by light and circadian oscillators. Neuroreport. 2002;13:2247-2251.

26. Kubo Y, Akiyama M, Fukada Y, Okano T. Molecular cloning, mRNA expression, and immunocytochemical localization of a putative blue-light photoreceptor CRY4 in the chicken pineal gland. $J$ Neurochem. 2006;97:1155-1165.

27. Yamamoto K, Okano T, Fukada Y. Chicken pineal Cry genes: light-dependent up-regulation of cCry1 and cCry2 transcripts. Neurosci Lett. 2001;313:13-16.

28. Berson DM. Phototransduction in ganglion-cell photoreceptors. Pflugers Arch. 2007;454:849-855.

29. Hardie RC, Raghu P. Visual transduction in Drosophila. Nature 2001;413:186-193.

30. Lee YJ, Shah S, Suzuki E, Zars T, O'Day PM, Hyde DR. The Drosophila dgq gene encodes a $G$ alpha protein that mediates phototransduction. Neuron. 1994;13:1143-1157.

31. Montell C. Visual transduction in Drosophila. Annu Rev Cell Dev Biol. 1999;15:231-268.

32. Hardie RC. TRP channels in Drosophila photoreceptors: the lipid connection. Cell Calcium. 2003;33:385-393.

33. Sekaran S, Foster RG, Lucas RJ, Hankins MW. Calcium imaging reveals a network of intrinsically light-sensitive inner-retinal neurons. Curr Biol. 2003;13:1290-1298.

34. Graham DM, Wong KY, Shapiro P, Frederick C, Pattabiraman K, Berson DM. Melanopsin ganglion cells use a membrane-associated rhabdomeric phototransduction cascade. J Neurophysiol. 2008; 99:2522-2532.

35. LaLonde MM, Janssens H, Rosenbaum E, et al. Regulation of phototransduction responsiveness and retinal degeneration by a phospholipase D-generated signaling lipid. J Cell Biol. 2005;169:471479.

36. Suh BC, Inoue $\mathrm{T}$, Meyer $\mathrm{T}$, Hille $\mathrm{B}$. Rapid chemically induced changes of PtdIns(4,5)P2 gate KCNQ ion channels. Science. 2006; 314:1454-1457.

37. Brocco MA, Panzetta P. Survival and differentiation of purified embryonic chick retinal ganglion cells cultured at low density in a chemically defined medium. J Neurosci Metbods. 1997;75:15-20.

38. Garbarino-Pico E, Carpentieri AR, Contin MA, et al. Retinal ganglion cells are autonomous circadian oscillators synthesizing Nacetylserotonin during the day. J Biol Chem. 2004;279:5117251181.

39. Bobu C, Craft CM, Masson-Pevet M, Hicks D. Photoreceptor organization and rhythmic phagocytosis in the Nile rat Arvicanthis ansorgei: a novel diurnal rodent model for the study of cone pathophysiology. Invest Ophthalmol Vis Sci. 2006;47:3109-3118.

40. Bird IM. Phosphoinositidase $\mathrm{C}$ activation assay. I Cell labeling, stimulation, and recovery of cellular $[3 \mathrm{H}]$ phosphoinositides and [3H]phosphoinositols. Methods Mol Biol. 1998;105:1-9.

41. Bird IM. Phosphoinositidase $\mathrm{C}$ activation assay. II. Simple analysis of recovered cellular phosphoinositides and phosphoinositols. Methods Mol Biol. 1998;105:11-23.

42. Bird IM. Preparation of $[3 \mathrm{H}]$ phosphoinositol standards and conversion of $[3 \mathrm{H}]$ phosphoinositides to $[3 \mathrm{H}]$ phosphoinositols. Methods Mol Biol. 1998;105:65-76.

43. Bligh EG, Dyer WJ. A rapid method of total lipid extraction and purification. Can J Biochem Pbysiol. 1959;37:911-917.
44. Jolles J, Bar PR, Gispen WH. Modulation of brain polyphosphoinositide metabolism by ACTH and beta-endorphin: structure-activity studies. Brain Res. 1981;224:315-326.

45. Folch J, Lees M, Sloane Stanley GH. A simple method for the isolation and purification of total lipides from animal tissues. $\mathrm{J} \mathrm{Biol}$ Chem. 1957;226:497-509.

46. Baldridge WH, Kurennyi DE, Barnes S. Calcium-sensitive calcium influx in photoreceptor inner segments. J Neurophysiol. 1998;79: 3012-3018.

47. Chomczynski P, Sacchi N. Single-step method of RNA isolation by acid guanidinium thiocyanate-phenol-chloroform extraction. Anal Biochem. 1987;162:156-159.

48. Agranoff BW, Fisher SK. Inositol, lithium, and the brain. Psychopharmacol Bull. 2001;35:5-18.

49. Hardie RC, Martin F, Cochrane GW, Juusola M, Georgiev P, Raghu P. Molecular basis of amplification in Drosophila phototransduction: roles for $\mathrm{G}$ protein, phospholipase $\mathrm{C}$, and diacylglycerol kinase. Neuron. 2002;36:689-701

50. Zuker CS. The biology of vision of Drosophila. Proc Natl Acad Sci USA. 1996;93:571-576.

51. Thanos S, Mey J. Development of the visual system of the chick, II: mechanisms of axonal guidance. Brain Res Brain Res Rev. 2001; 35:205-245.

52. Hartwick AT, Bramley JR, Yu J, et al. Light-evoked calcium responses of isolated melanopsin-expressing retinal ganglion cells. J Neurosci. 2007;27:13468-13480.

53. Wong KY, Dunn FA, Graham DM, Berson DM. Synaptic influences on rat ganglion-cell photoreceptors. J Physiol. 2007;582:279-296.

54. Schmidt TM, Kofuji P. Functional and morphological differences among intrinsically photosensitive retinal ganglion cells. J Neurosci. 2009;29:476-482

55. Fisher SK, Heacock AM, Agranoff BW. Inositol lipids and signal transduction in the nervous system: an update. I Neurochem. 1992;58:18-38.

56. Hardie RC. Phototransduction in Drosophila melanogaster. $J$ Exp Biol. 2001;204:3403-3409.

57. Goto K, Kondo H. Functional implications of the diacylglycerol kinase family. Adv Enzyme Regul. 2004;44:187-199.

58. Sakane F, Imai S, Yamada $\mathrm{K}$, Kanoh $\mathrm{H}$. The regulatory role of EF-hand motifs of pig $80 \mathrm{~K}$ diacylglycerol kinase as assessed using truncation and deletion mutants. Biochem Biophys Res Commun. 1991;181:1015-1021.

59. Kai M, Sakane F, Imai S, Wada I, Kanoh H. Molecular cloning of a diacylglycerol kinase isozyme predominantly expressed in human retina with a truncated and inactive enzyme expression in most other human cells. J Biol Chem. 1994;269:18492-18498.

60. Giusto NM, Ilincheta de Boschero MG. Synthesis of polyphosphoinositides in vertebrate photoreceptor membranes. Biochim Biophys Acta. 1986;877:440 - 446.

61. Huang Z, Ghalayini A, Guo XX, Alvarez KM, Anderson RE. Lightmediated activation of diacylglycerol kinase in rat and bovine rod outer segments. J Neurochem. 2000;75:355-362.

62. Wu L, Niemeyer B, Colley N, Socolich M, Zuker CS. Regulation of PLC-mediated signalling in vivo by CDP-diacylglycerol synthase. Nature. 1995;373:216-222.

63. Harris WA, Stark WS. Hereditary retinal degeneration in Drosophila melanogaster: a mutant defect associated with the phototransduction process. J Gen Physiol. 1977;69:261-291. 